\title{
MedienPädagogik
}

www. medienpaed.com

Zeitschrift für

Theorie und Praxis

der Medienbildung

ISSN 1424-3636

Themenheft Nr. 22: Frühe Medienbildung

\section{Medienpädagogik in der frühen Kindheit? Pädagogische Anmerkungen zur Normalisierung eines neuen Bildungsbereiches}

\author{
Burkhard Fuhs
}

\begin{abstract}
Im folgenden Beitrag soll der Bildungsbereich der Medienpädagogik in der frühen Kindheit angesichts des defizitären Bilds auf eine kindliche Mediennutzung diskutiert werden. Der Frühpädagogik kommt aufgrund der Mediatisierung der kindlichen Lebenswelt die Aufgabe zu, Medien unter Berücksichtigung der Anforderungen dieses spezifischen pädagogischen Bereichs in die pädagogische Arbeit zu integrieren.
\end{abstract}

\section{Neue Bildungsorientierungen}

Medienpädagogik und Frühpädagogik stehen in einem Spannungsverhältnis, das sich im Kontext unterschiedlicher Traditionen, Werte und Normen und als Ausdruck differenter Fachdisziplinen und pädagogischer Selbstverständnisse entwickelt hat. Die Veränderungen von Kindheit, insbesondere in den ersten sechs Jahren, die Mediatisierung sowie die auf Sachlernen abgestellte Bildungsorientierung, stellen eine grosse Herausforderung dar und bieten die Chance, über Medienbildung und Frühpädagogik neu nachzudenken.

Über Medienpädagogik in der frühen Kindheit zu reflektieren bedeutet zunächst einmal, die ersten Lebensjahre der Kinder selbst in den Blick zu nehmen. Was sind die Rahmenbedingungen, Voraussetzungen und Zielvorgaben, unter denen im Alter von 0-6 Jahren Medienerziehung, Medienbildung, Mediensozialisation bzw. Medienkompetenzvermittlung stattfinden, stattfinden können und sollen? Welche Fähigkeiten bringen Kinder, Eltern, Pädagogen und Pädagoginnen in den Umgang mit Medien ein? Welche Interessen, Erfahrungen, Hoffnungen und Befürchtungen begleiten den Prozess der Etablierung einer neuen Medienpädagogik in der Zeit vor der Schule?

Schon diese Aufzählungen zeigen ein hohes Mass an Komplexität und Multidisziplinarität, die eine Pädagogik mit Medien in der frühen Kindheit in Theorie und Praxis herausfordern: Entwicklungspsychologie, Hirnforschung, Sozialpädagogik, empirische Bildungsforschung, Frühpädagogik, Medienwissenschaft, Soziologie und Politik sind wichtige Bezugswissenschaften für ein grundlegendes Verständnis der Rahmenbedingungen unter denen Medienpädagogen/innen zukünftig in einem Feld agieren wollen (und sollen), das aber vor dem Hintergrund ihrer (Praxis-) Tradition und ihrer bisherigen (theoretischen) Ausbildung eher fremd und als nicht in ihren Zuständigkeitsbereich gehörend wahrgenommen wurde und wird.

Fuhs, Burkhard. 2015. «Medienpädagogik in der frühen Kindheit? Pädagogische Anmerkungen zur Normalisierung eines neuen Bildungsbereiches» MedienPädagogik 22 (11. Mai): 1-14. https://doi.org/10.21240/mpaed/22/2015.05.11.X. 
Der Bereich der frühen Kindheit hat sich in den letzten Jahren tief greifend verändert, und der Prozess einer neuen Bildungsorientierung der frühen Pädagogik, einer Neustrukturierung der Kinderinstitutionen der ersten Lebensjahre, einer Professionalisierung der Einrichtungen und einer Qualitätskontrolle durch umfassende Evaluation ist noch lange nicht abgeschlossen. Dieser Wandel bietet neue Chancen für Kinder, Eltern und Erzieherlnnen, verunsichert aber auch viele Menschen, die in diesem Praxisfeld Verantwortung tragen. Durch die Bildungspläne, die in allen Bundesländern entstanden sind, wird deutlich, dass zukünftig die pädagogische Qualität der Praxis in den Familien, in den Kitas, in den ausserschulischen Handlungsfeldern, im Hortbereich und natürlich auch in der Schule vor allem an der Bildungsqualität der jeweiligen Massnahmen gemessen werden wird.

\section{Krise der Frühpädagogik}

Noch vor zehn Jahren konstatierte Wassilios E. Fthenakis in einer Publikation, die sich mit der Frage auseinandersetzte, "wie aus Kindertagesstätten Bildungseinrichtungen werden können» (Fthenakis 2006) eine tief greifende Krise der Frühpädagogik. Es lässt sich jedoch ein Prozess der Neuverortung und Neuorientierung der Pädagogik in den Kindergärten, -krippen und -horten beobachten, der seit den 1990er-Jahren verstärkt in Bewegung gekommen ist (Fthenakis u. Textor 2000). Fthenakis stellt fest, dass die «sehr innovativen Ansätze» der 1970er-Jahre nicht umgesetzt wurden: «Wir stehen heute vor einer vergleichbaren Situation wie vor dreissig Jahren. Im Unterschied zu den siebziger Jahren kommt jedoch diesmal die Forderung von unterschiedlichen Seiten, und sie ist stärker als je zuvor auf die Bildung und Erziehung von Kindern unter sechs Jahren fokussiert» (Fthenakis 2006, 10).

Fragt man nach dem Motor für den grundlegenden Perspektivenwechsel in der Frühpädagogik, so finden sich zahlreiche Hinweise dafür, dass die weltweiten wirtschaftlichen Veränderungen den Prozess angestossen haben und in Gang halten. So betonen Toni Mayr und Michaela Ulich in ihrer pädagogischen Analyse des Konzeptes «Engagiertheit von Kindern» als Grundlage eines neuen Kita-Modells die engen Verbindungen von Wirtschaft und Frühpädagogik und die selbstverständliche Notwendigkeit der Erziehungswissenschaft, den Anschluss an die ökonomische Argumentation zu suchen: «Ausgehend von der Wirtschaft hat die Diskussion um Qualität und Qualitätssicherung auf viele andere Bereiche übergegriffen und schliesslich auch die Pädagogik erreicht. Als wichtiger Bestandteil der pädagogischen Landschaft und als erste Stufe des Bildungssystems muss sich selbstverständlich auch die Elementarerziehung dieser Diskussion stellen» (Mayr u. Ulich 2003, 169).

«Das Bildungssystem der Bundesrepublik» - Hans-Joachim Laewen 2001 - «wird zurzeit aufgrund grosser Herausforderungen durch die globalisierte Wirtschafts- 
verflechtung und die sich daraus ergebenden Anforderungen an die Qualifizierung der Fachkräfte grundlegend reformiert. «Mehr Bildung` wird vom Präsidenten der deutschen Arbeitgeberverbände als Bedingung für die Bewältigung zukünftiger Aufgaben gefordert» (Laewen 2007, 35).

Auch die Bildungsinitiative der Unternehmensberatung McKinsey \& Company zeigt das hohe Engagement der Wirtschaft für die frühe Bildung. «Mit dem zweiten Bildungskongress», meldete 2005 der FOCUS, «endete gestern in Berlin die Initiative «McKinsey bildet〉. Unter dem Motto «Wer an den Kindern spart, wird in der Zukunft verarmens trafen sich Experten seit Januar 2005 in vier Werkstattgesprächen. Thema waren vor allem die Defizite bei der frühkindlichen Bildung» (Sanktjohanser 2005). Die Qualitätsentwicklung und Qualitätssicherung in der Frühpädagogik, die entlang ausgefeilter Indikatorenlisten umgesetzt und am Lernprozess der Kinder angesetzt wird, kann also als Teil eines gesamtgesellschaftlichen Controllings verstanden werden, bei dem die Kriterien für den Erfolg nicht mehr alleine intern von den Akteuren in einem Feld festgelegt werden, sondern Ausdruck eines gesellschaftlichen Normierungsprozesses sind, der sich vor allem an der Zukunftsfähigkeit der Wirtschaft orientieren soll und muss.

\section{Naturwissenschaftliche Frühförderung}

Das Interesse der Wirtschaft an der frühen Bildung wird an vielen Stellen sichtbar, etwa wenn Industrieverbände wie SÜDWESTMETALL eine Initiative zur Frühförderung unterstützt. Das Programm zeigt den Versuch, in den ersten Lebensjahren eine naturwissenschaftlich-technische Bildungsoffensive, wirtschaftliche Belange und den erwarteten zukünftigen Fachkräftebedarf mit den Anforderungen einer neuen Elementarbildung in Einklang zu bringen: «Technolino fördert durch die Einrichtung von Forscherecken in Kindergärten den spielerischen Umgang mit Naturwissenschaft und Technik und das Interesse an naturwissenschaftlich-technischen Phänomenen. Diese technischen Inhalte werden mit Sprach-, Kreativitätsund Bewegungserziehung sowie ethischer und musikalischer Erziehung verknüpft» (SÜDWESTMETALL.de). Verständlich wird dieses unternehmerische Engagement für die Pädagogik, wenn man die Bedeutung von Bildung, Ausbildung und Weiterbildung für den nationalen Bestand der deutschen Industrie angesichts der kurzen Innovationszyklen und einer scharfen weltweiten Marktkonkurrenz in den Blick nimmt: «Die Bildungspolitik ist eine wichtige Säule von SÜDWESTMETALL. Denn nur mit qualifizierten Mitarbeitern bleibt unsere Industrie zukunftsfähig» (ebd.). Diesem ausgewählten Beispiel könnten ohne Schwierigkeiten weitere aus anderen Branchen und Bundesländern an die Seite gestellt werden.

Diese Wirtschafts- und Zukunftsorientierung im Prozess der Reform der Frühpädagogik ist heute nicht mehr so deutlich spürbar wie vor zehn Jahren, sie bestimmt aber weiterhin die Entscheidungen und Investitionen in diesem Bereich und dient 
gewissermassen als Werbeargument um Aufmerksamkeit und Engagement in diesem bisher wenig beachteten pädagogischen Bereich zu erhöhen.

Auf die Frage, warum eine «tief greifende Reform des Bildungsauftrags des Kindergartens» gefordert wird, führte Fthenakis $(2006,10)$ - vor knapp zehn Jahren - als Erstes den gesellschaftlichen Wandel und die Notwendigkeit für das Individuum an, Kompetenzen für die Bewältigung der neuen komplexen Lebensbedingungen auszubilden. Zweitens sieht er für die Zukunft die Notwendigkeit einer Multilingualität und einer interkulturellen Kompetenz. Drittens liefere die Wirtschaft «mit ihren neu definierten Anforderungen an das Individuum als zukünftige Arbeitskraft» wichtige Argumentationslinien für eine neue bildungsorientierte Frühpädagogik: Die Wirtschaft «erwartet nicht mehr bloss Wissen, sondern Kommunikationskompetenz, Teamfähigkeit, Mobilität, interkulturelle Kompetenz, die Fähigkeit, unter unterschiedlichen Umständen angemessene Entscheidungen zu treffen, Risikobereitschaft und vieles mehr. Dieses veränderte Profil an Erwartungen eröffnet erstmalig die Chance, ein Konzept von Bildung zu entwerfen, das nicht bloss eng auf die berufliche Vorbereitung des Kindes gerichtet ist, sondern auf Kompetenzerwerb für das ganze Leben. Die Forderung, die daraus abzuleiten ist, heisst dann: Stärkung kindlicher Kompetenzen, und zwar von Beginn des Lebens an» (BMFSFJ 2012) 1 .

Heute wird die Wirtschaftsorientierung der neuen Frühbildung nicht mehr in den Vordergrund gestellt. Vielmehr zeigen die neuen Programme, etwa die «Offensive Frühe Chancen» des Familienministeriums (ebd.) eine deutliche Orientierung an der sozialen Ungleichheit, die durch die neue Bildungsorientierung und die mehrten Bildungsanstrengungen von bildungsorientierten Eltern in der frühen Kindheit entstanden ist und sich zu vertiefen droht. Ohne dass diese Entwicklung benannt wird, setzt die Politik auf Korrekturmassnahmen: "Dies ist ein weiterer wichtiger Schritt» - so Kristina Schröder im März 2012 - «zu unserem Ziel: Alle Kinder sollen von Anfang an faire Chancen haben! In den Schwerpunkt-Kitas werden die Jüngsten ganzheitlich und systematisch in ihrer Entwicklung begleitet. So schaffen Sprachexperten vor Ort die Basis für eine erfolgreiche Bildungs- und Berufslaufbahn und die Integration in die Gesellschaft» (ebd.). Die Verbesserung der Zukunftschancen der deutschen Wirtschaft führt so auf der individuellen Ebene zu einem grösseren Risiko der Familien und Kinder, da nicht alle einen fairen Zugang zu den neuen Bildungschancen erhalten, da die neue frühkindliche Bildung mit hohen Kosten an Zeit und an Finanzen verbunden ist. Die hohen sozialen Unterschiede in den Bildungskarrieren der Kinder, die in den frühen Jahren in der Familie entstehen (Lange 2010), werden auch und insbesondere durch den Mediengebrauch der Heranwachsenden verstärkt (Theunert 2010).

1 BMFSFJ 2012: [online] http://www.bmfsfj.de/BMFSFJ/kinder-und-jugend,did=185538.html (27.4.2012). 


\section{Frühpädagogischer Aufbruch ohne Medien?}

Für die Medienpädagogik in der frühen Kindheit ist von entscheidender Bedeutung, dass die wirtschaftliche Zukunft, für die der Elementarbereich vorbereiten soll, im Kontext der Bildungsinitiativen in scharfer Abgrenzung zur Medienwelt konstruiert wird. Ganz in der pädagogischen Tradition des Warnens und Mahnens vor einer Wirklichkeit aus zweiter Hand (Bauer u. Hengst 1980) sind frühpädagogische Konzepte vor allem auf die sinnlichen Erfahrungen beim Tun, beim Umgang mit Sachen und beim Handeln in der gegenständlichen Lebenswelt konzentriert. Die Physik- und Chemiekoffer zeigen diese haptische Orientierung ebenso wie die Bildungspläne. Die Angst vor der Medienwelt als der schlechtesten aller Bildungsmöglichkeiten wird auch von der Hirnforschung und den von ihr entworfenen Schreckensbildern geschürt. Sie findet sich in einer Theorie der Zweiteilung der Welt in primäre und sekundäre Welterfahrung, wie sie etwa im Thüringer Bildungsplan entworfen wird (Thüringer Ministerium für Bildung, Wissenschaft und Kultur 2010).

«Kinder [sammeln] Primärerfahrungen, insbesondere im Umgang mit Naturmaterialien sowie im Umgang mit interessanten Alltagsgegenständen [...]. Kinder [sammeln] Sekundärerfahrungen, indem sie sich nicht nur mit Originalen, sondern auch mit Reproduktionen in Kunstkatalogen und Kunstbänden, in Bilderbüchern sowie auf Postern [...] auseinandersetzen» (ebd., 132). Diese Unterscheidung wird nicht nur für das künstlerische Lernen gemacht. Viele Bildungsbereiche im Bildungsplan kommen ganz ohne Medien aus und nur am Rande werden etwa Bilderbücher erwähnt. Medien erscheinen als Problembereich, den es zugunsten eines Hantierens mit Materialien zu vermeiden gilt. «Die 〈Medienkindheit»» - so der Thüringer Bildungsplan - «erfordert die Unterscheidung von selbst gemachten Erfahrungen (primäre Bildungserfahrungen) und durch Medien vermittelte Erfahrungen (sekundäre Bildungserfahrungen). Deshalb ist es wichtig, einen kritischen Umgang mit Medien und deren Inhalten zu erwerben» (ebd., 139).

Daniel Süss, Claudia Lampert und Christine W. Wijnen haben sich in ihrer Einführung zur Medienpädagogik sehr kritisch mit dem Argument der Medienerfahrungen als Sekundärerfahrungen auseinandergesetzt und wehren sich gegen eine Abwertung der Medienerfahrungen als «wertlos»: Nicht die Form der Realität sei wichtig, sondern ob eine Lebenswelt anregungsarm und anregungsreich sei und sowohl die Alpha-Welt als auch die Beta-Welt können für die Bildung von Kindern reichhaltig oder einseitig sein (Süss et al. 2010, 19).

Vielmehr seien die Medien eine neue grundlegende Herausforderung für unsere Gesellschaft und das Lernen mit Bildern, Worten oder Musik gehöre zur Alphabetisierung in unserer Kultur zwingend dazu (ebd., 22). Die Mediatisierung der Lebenswelt hat den Umgang mit Medien zu einer primären Erfahrung aller Kinder mit der 
Welt gemacht und Medienbildung, Medienerziehung und Medienkompetenz gehören heute zum Leben von Geburt an dazu wie die Medien auch (Theunert 2007). Die strikte Zweiteilung der Welt in reale Welt und Medienwelt, wie sie die Frühpädagogik in Teilen macht, zeigt also, dass Erkenntnisse der Medienpädagogik nicht genügend in die Bildungsorientierung des Elementarbereiches Eingang gefunden haben. Menschen als symbolische Wesen haben es in ihren Kommunikationsprozessen eher mit einer Bedeutungskonvergenz zu tun als mit festen Grenzen zwischen den unterschiedlichen Realitätsmodi. Die Zweiteilung geht weit an den Spiel- und Fantasiewelten vorbei und unterschätzt die Kinder als realitätsverarbeitende Akteure vollständig.

\section{Die neue Bildungsorientierung als Sachlernen}

Als weiteres Problem kommt hinzu, dass in der Wende der Frühpädagogik zur Bildung eine Überbetonung des Sachlernens eingeschlossen ist. Die Theorie von primären Erfahrungen mit der Welt, die als Hantieren mit Sachen gefasst wird, ist ein direkter Ausdruck einer deutlichen Orientierung am naturwissenschaftlichen Weltbild, an der technisch-mathematischen Bildung und einem sozialen Lernen, das an «realen» Beziehungen orientiert ist. Die Sachorientierung und der Boom des Sachlernens, die die Bildungspläne durchziehen, führen zu endlosen Listen von Wissensbeständen. Während in der Theorie Kompetenzen als Fähigkeiten gelingenden Handelns in definierten Praxisbereichen entworfen werden, müssen in den Kinderinstitutionen nicht selten Themen-, Sach- und Erfahrungsbereiche abgearbeitet werden.

\section{Neue Qualitätsstandards in der Bildung}

Die neuen Qualitätsstandards definieren die Strukturen, Inhalte, Beziehungen, Prozesse, Kompetenzziele und Kontrollverfahren für die Frühpädagogik und sind von einem Professionalisierungsprozess begleitet, der zu einem veränderten Berufsbild und einem forcierten Aus- und Weiterbildungsbedarf bei ErzieherInnen geführt hat (Ballusek 2008). In diesem Kontext bekommt die empirische Forschung in der Praxis einen neuen Stellenwert für die Qualitätssicherung, und Erzieherinnen und Erzieher sind gefordert, die Bildungsprozesse in ihren Einrichtungen sichtbar und überprüfbar zu machen. Es kommen eine Vielzahl von qualitativen und quantitativen Methoden zum Einsatz. Auch der enge Dialog mit den Eltern als Bildungspartner soll in den Evaluationsprozess eingebunden werden (Beber 2008). Dass die Skalen zur «zuverlässigen Erfassung der Qualität von pädagogischen Prozessen» in Krippen, Kindergärten und Horten (Umgang mit Kindern, Anregungen, Alltag, Interaktionen mit Erwachsenen und Kindern, altersgerechte Gestaltung) aus dem Englischen übernommen wurden, zeigt zum einen die internationale Vernetzung 
des Prozesses und zum anderen den globalen Reformierungsdruck, der auf der frühen Kindheit liegt (Tietze u.a. 2007).

Die Neuorientierung der Frühpädagogik hat nicht nur zu einer neuen Kultur der Anregung der Kinder geführt, sie entwirft ein neues Kindheitsbild und betont die sozial eingebundene Eigenaktivität der Kinder im Bildungsprozess. Die Rolle der Erzieher/innen und Eltern wird nicht mehr in einer situativen Begleitung der Kinder verstanden, sondern in einer Ko-Konstruktion. Die Kinder werden nicht als isolierte Lernende gesehen, sondern als Lernende in einem Netz aus anderen Kindern und vor allem Erwachsenen, die durchaus Anregungen und Korrekturen geben sollen und können. Dass Kinder zum einen als Lernende auch im Alter von unter sechs Jahren ernst genommen werden, zeigen Label wie «Forscher, Künstler, Konstrukteure» (Laewen 2007). Gleichzeitig wird aber auch die Zukunftsorientierung der neuen Frühpädagogik deutlich, die sich vom einfachen kindlichen Spiel abgrenzt und in den Zuschreibungen auch Erwartungen an die Kinder als zukünftige Wissenschaftler, Kulturschaffende und Ingenieure formuliert. Kinder wollen lernen, sie können lernen und sie tun dies besonders gut, wenn sie angeregt werden und das Lernen ihnen Spass macht. Dies ist eine der Grundbotschaften der frühen Förderungen von Kindern (ebd., 10ff.), die sich expliziert auch auf die neuen Erkenntnisse der Hirnforschung (Singer 2003) bezieht und Lernformen, die nicht auf Lust, Neugier und Selbstwirksamkeit zielen, als wenig wirksam herausstellt: «Der Welterkundungsdrang von Kindern, so die ernüchternde Erkenntnis [...], wird im durchschnittlichen deutschen Kindergarten oft nicht nur ignoriert, sondern sogar behindert. Was Kinder umtreibt, was in ihren Köpfen vorgeht, wie und was sie lernen - davon haben die Menschen, die von Berufs wegen täglich mit ihnen umgehen, viel zu wenig Ahnung» (Laewen 2007, 25).

Bildung in der frühen Kindheit wird damit konsequent auf das Lernen und die Tätigkeiten der Kinder bezogen. Frühe Bildung kann dabei als eine Pädagogik verstanden werden, die einen aktiven, sich selbstbildenden Bildungsbegriff in den Mittelpunkt stellt: «Wenn Bildung die zentrale Aktivität bezeichnet, über die Kinder sich die Welt aneignen - eine innere Welt konstruieren [...] -, dann kann ein Kind nicht gebildet werden, es kann sich nur selbst bilden» (Laewen 2007, 42). Aber die Kinder bilden sich nicht in sozialer Isolation.

Mit Ko-Konstruktion ist ein pädagogischer Ansatz umschrieben, der das gemeinsame Handeln zwischen Kindern und Erzieher/innen in den Mittelpunkt stellt. «Der Schlüssel der Ko-Konstruktion ist die soziale Interaktion, sie fördert die geistige, sprachliche und soziale Entwicklung. Das Kind lernt, indem es seine eigenen Ideen und sein Verständnis von der Welt zum Ausdruck bringt, sich mit anderen austauscht und Bedeutungen aushandelt. Der reine Erwerb der Fakten tritt somit in den Hintergrund. Das Kind besitzt demnach eigene Ideen und Theorien, denen es sich lohnt zuzuhören, die aber auch infrage gestellt werden können» (Bayerisches 
Staatsministerium für Arbeit und Sozialordnung, Familie und Frauen, Staatsinstitut für Frühpädagogik 2006, 427).

\section{Anschlussfähigkeit: Spielen oder was Richtiges lernen?}

Entscheidend ist die Frage, ob, wann und wie selbst gewählte kindliche Lernprozesse unter- oder abgebrochen werden zugunsten «qualitätvoller» Inhalte aus Erwachsenensicht. Während in der traditionellen Frühpädagogik die Lernprozesse der Kinder und das Interesse der Kinder in der Gefahr standen, nicht ernst genommen zu werden und unter dem Begriff des Spielens subsumiert und ausgeblendet zu werden, besteht nun die Gefahr, dass das kindliche Spiel den adultistischen Bildungszwängen zum Opfer fällt. In ihrer Geschichte haben pädagogische Einrichtungen immer wieder das kindliche Spielen gegenüber den «richtigen» Tätigkeiten abgewertet (Fuhs 1997). Dass nach PISA mit der neuen «ernsthaften s fühen Bildung das Erzählen und die kindliche Fantasie zurückgedrängt wurden, zeigt sich an unterschiedlichen Stellen: Die hohe Bedeutung, die etwa die Sachliteratur in der frühen Bildung hat, zeigt die pädagogische Bemühung, die Kinder möglichst früh in die rationale Welt der Erwachsenen einzuführen (Börsenverein des Deutschen Buchhandels in Zusammenarbeit mit der Arbeitsgemeinschaft von Jugendbuchverlagen e. V. 2007). Das Beratungsportal Familie.de beschreibt die frühe Kindheit als eine Entwicklung zur Rationalität: «Zwischen vier und sechs Jahren wandelt sich die Weltsicht der Kinder nachhaltig. Sie wachsen aus der magischen Phase heraus und entwickeln nach und nach einen - wenn auch noch naiven - Realitätssinn» (Familie.de, 25.4.2012). Aufschlussreich für die Einstellung der Eltern zu diesem Prozess ist die Warnung des Ratgeberportals, die Kinder nicht vorschnell aus ihrer Fantasiewelt zu drängen. Das Portal zeigt ein Argumentationsmuster, das häufiger anzutreffen ist:

Im Vorschulalter entwickeln Kinder übrigens auch die ersten Spezialinteressen, schmökern in Sachbüchern und wollen alles über Ritter, Dinosaurier, Pferde oder Eisenbahnen wissen. Aber schon einen Moment später befinden sie sich wieder in ihrer magischen Gedankenwelt und verstehen vieles doch noch nicht. «In solchen Momenten überfordern wir unsere kleinen Schlauberger leicht, weil wir bereits ein logisches Denken und planvolles Handeln von ihnen fordern, das sie gar nicht leisten können», sagt Daniela Liebich. (ebd.)

Festhalten lässt sich, dass die neue Bildungsorientierung in der Frühpädagogik eng mit einer Betonung von Sachkultur und von Fakten einhergeht, die nicht selten «kindgerecht» in kleine motivierende Erzählungen eingefüllt werden. Die Bildungspläne der Bundesländer zeigen deutlich in den Themen die Dominanz der Sachkultur, die als aktive Aneignung der (Erwachsenen-) Welt durch die Kinder gedacht wird. Die Vielzahl der Themen, die in den ersten sechs Jahren behandelt 
werden sollen, ist dabei so umfangreich, dass der Plan nur mit Anstrengungen umzusetzen ist.

\section{Neue Ungleichheiten und die Eigenlogik der Kinderwelt}

Die hohe Wissens- und Kompetenzorientierung in der frühen Bildung führt unweigerlich zu einem neuen gesellschaftlichen Bereich von Ungerechtigkeit und sozialer Ungleichheit, da die notwendige Mitarbeit der Eltern, die bestehenden und weiterwirkenden Ungleichheiten des Bildungserwerbs in der Familie (Büchner u. Brake 2006) vermutlich noch verstärken wird. Dass das Sachlernen gegenüber einem narrativen Lernen durch Medien überbetont wird, könnte ein weiteres Moment sein, bildungsferne Kinder zu benachteiligen, da es vor allem bildungsferne Kinder sind, die eher narrative Medienformate wählen (Niesyto 2012). Diese narrative Struktur der frühen Kindheit ist relativ gut erforscht (Paus-Haase 1997) und Horst Niesyto fordert für eine milieusensible Medienpädagogik gerade die pädagogische Arbeit an den ästhetischen Medienerfahrungen der Heranwachsenden, um benachteiligten Kinder neue Chancen zu eröffnen (ebd., 154).

Während die Bildungswelt sich in der frühen Kindheit aus einer komplexen Struktur von Inhalten mit Anschluss an die Erwachsenen zusammensetzt, bewegen sich die Kinder in eigenen Spiel- und Fantasiewelten, in denen sie grundlegende Entwicklungsaufgaben bearbeiten. Stets sind es bestimmte Probleme, die die Kinder fordern (Schäfer 2005) und zur Beschäftigung mit Themen führen, die auch die Medienauswahl bestimmen. Dies bedeutet nicht, dass die Frühpädagogik im Sinne einer Ko-Konstruktion, die Bildungsprozesse der Kinder nicht anregen oder begleiten dürfte.

Die Medienwelten der Kinder sind Bereiche, die die Kinder in hohem Masse faszinieren, sie sind nicht logisch, systematisch aufgebaut, sondern eher narrativ, auf Abenteuer, auf Helden und auf Identifikation ausgelegt. Märchen, Kinderliteratur, Computerspiele, Hörspiele, Bilder, Kinderfilme, Reime, Lieder, Bilderbücher zeugen zum einen von einer reichen Kindermedienwelt, die die Kindheit seit den letzten Jahrhunderten begleitet und ausgemacht hat. Sie mahnen zum anderen, die ästhetische Bildung und die Ausbildung der inneren Welten der Kinder, der Fantasie und der Beschäftigung mit all den Dingen, die den Kindern im Spiel, beim Betrachten von Bildern, beim Lesen von Geschichten, beim Fernsehen und Hörspielhören durch den Kopf gehen, nicht zu vernachlässigen. Die ästhetische Bildung der Kinder, die Ermöglichung ästhetischer Erfahrungen, ist für die grundlegende Bildung das allerwichtigste pädagogische Angebot, weil sie an der zentralen Welterfahrung der Kinder ansetzt (Mollenhauer 1996) und eine Basis für alle weiteren Bildungsprozesse bietet. Dieser intermediäre Raum (Winnicott 2005) der Kindermedien ist ebenso wichtig wie das Spielen und die Bildungsbuchforschung 
hat deutlich den Wert einer frühen Begegnung mit hochqualitativen Medien für Kinder herausgearbeitet (Thiele 2007).

\section{Medienpädagogik und frühe Bildung}

Die neue propagierte Zukunftsorientierung der frühen Kindheit findet pädagogisch unter Ausschluss der Medien statt, die als tendenziell problematisch beschrieben werden, auch wenn anerkannt wird, dass im Medienlernen auch Chancen eingeschlossen sind (ebd.). Lernen mit Medien im Elementarbereich wird so vor allem unter Jugendschutzfragen behandelt (vgl. Grussworte in Theunert 2007); und Elternratgeber warnen die Erziehungsberechtigten eindringlich davor, ihren Kindern zu früh und unkontrolliert den Zugang zu Medien zu erlauben. Die Eskamotage der Medien aus den pädagogisch kontrollierten Bereichen der Frühpädagogik spiegelt also eine grundlegende Angst in unserer Kultur vor dem Einfluss der Medien, die eine planvolle Erziehung und zukunftsorientierte Bildung behindern und verhindern könnten und die in der Lage seien, den Kindern zu schaden. Die Einleitung des christlichen Ratgebers «Kinder in der Mediengesellschaft» macht den Konflikt und die Befürchtungen deutlich: «Würden Sie Ihr Vorschulkind alleine auf den Strassenverkehr loslassen? Oder Ihr Grundschulkind ohne Begleitung in die nächste Grossstadt schicken? [...] Genauso wie Kinder das richtige Verhalten im Strassenverkehr lernen müssen, benötigen sie auch Hilfe, Wegweisung und klare Regeln mit den Medien» (Nieswiodek-Martin 2006, 8).

Diese suggestiven Fragen konstruieren eine Welt, in der eine direkte Partizipation der Vorschulkinder an der Welt der Erwachsenen als Gefahrenpotenzial heraufgeschworen wird. Die Notwendigkeit eines Jugendmedienschutzes ist aber kein Argument für die Verbannung von Medien aus der Frühpädagogik. Es stellt sich vielmehr die Frage nach der kindgerechten Gestaltung der Lebenswelt der Kinder. Der Vergleich von Medien und Verkehr ist bei Nieswiodek-Martin nicht nur zu kurz gegriffen, sondern verschleiert die pädagogische Verantwortung, um die Medien zu diskreditieren.

Die Medienpädagogik tut sich mit den neuen Bildungsanforderungen in der frühen Kindheit schwer. Solange adultistische Voraussetzungen für die Medienbildung formuliert werden, bleibt eine frühkindliche Medienbildung in einem Defizitbild von Kindern stecken, denen nur eine rudimentäre Medienkompetenz vermittelt werden kann.

Dass Medien Gegenstand von frühkindlicher Bildung und gleichzeitig frühkindliche Bildung Inhalt von Medien sind, lässt sich zwar an den neuen Angeboten im Internet und Fernsehen für den Vorschulbereich ablesen. Aber immer noch hängt die Medienpädagogik an einem Medienkompetenzbegriff, der sich an Jugendliche und Erwachsene wendet und einen Emanzipationsprozess durch Aufklärung in Gang setzen möchte, der nur schwer auf den Elementarbereich zu übertragen ist. 
Die Kompetenzbereiche und das Medienwissen, über deren Vermittlung sich die Disziplin definiert, sind nicht mit der frühen Bildung kompatibel: «Die im vorherigen Abschnitt zusammengefassten Ziele der Medienerziehung mögen den Eindruck einer völligen Überforderung all jener erwecken, die sich um eine praktische Umsetzung medianpädagogischer Ziele bemühen» (Six u. Gimmler 2007, 27).

Bisher hat die neue Frühbildung ebenso wenig die neuen Erkenntnisse und Ansätze der Medienbildung aufgenommen und in ihren pädagogischen Bereich transformiert, wie die Medienpädagogik die neuen Lernformen und die Bildungsorientierung im Elementarbereich wahr- und ernst genommen hat. Die Medien in der pädagogischen Arbeit auszugrenzen ist angesichts einer durchgreifenden Mediatisierung der frühen Kindheit ebenso wenig zielführend, wie die Übertragung medienpädagogischer Ansätze und Ziele auf den Bereich der frühen Kindheit, ohne die besonderen Anforderungen dieses Bereiches und die Notwendigkeit eigener Lern- und Bildungsformen für den Vorschulbereich anzuerkennen.

\section{Fazit}

Festzuhalten bleibt es Auftrag für die Medienpädagogik, dass die Verbindung der inneren und äusseren Welt der Kinder ein zentraler Fokus aller Bemühungen in der frühen Kindheit sein müssen und einen zentralen Stellenwert in der pädagogischen Arbeit erhalten müssen.

Medienbildung als Form der Verbeheimatung in der mediatisierten Lebenswelt muss als Erlernen der Mediennutzung und Lernen mit Medien verstanden werden und braucht neue Fundierung in der frühen Kindheit, die die neueren Ansätze und Herausforderungen aufgreift und eine eigensinnige Medienpädagogik für den Elementarbereich entwickelt, die bei Kindern, Eltern, Pädagogen und einer kritischen Öffentlichkeit Bestand hat. Lernen mit Medien ist dabei als eine ganz eigene Bildungsform anzusehen, die anderen Gesetzen folgt als etwa die Schulbildung oder die bisherigen Lernformen in den Kitas. Es ist keineswegs eine Aufgabe allein für die Familien, wie die Medienpädagogik manchmal behauptet: «Im frühen Kindesalter finden diese Prozesse der Mediensozialisation fast ausschliesslich in der Familie statt» (Spanhel 2006, 113). Viel zu vernetzt sind Familien heute, zu gross ist die Bedeutung der Betreuung von Kindern, zu wichtig sind die Angebote der Medienschaffenden, die etwa im öffentlich-rechtlichen Fernsehbereich ihre Aufgabe eines kindgerechten Bildungsangebotes längst erkannt und angenommen haben (etwa das Vorschulangebot des Kinderkanals Kikaninchen), ebenso wie auch die privaten Sender deutlich auf Elementarbildung setzen. In der Medienpädagogik für die frühe Kindheit ist noch viel Luft nach oben, für Theorie und Praxis. 


\section{Literatur}

Balluseck, Hilde von, Hrsg. 2008. Professionalisierung der Frühpädagogik. Perspektiven, Entwicklungen, Herausforderungen. Opladen, Farmington Hills: Barbara Budrich.

Bauer, Karl W. und Heinz Hengst. 1980. Wirklichkeit aus zweiter Hand: Kindheit in der Erfahrungswelt von Spielwaren und Medienprodukten. Reinbek bei Hamburg: Rowohlt Verlag.

Bayerisches Staatsministerium für Arbeit und Sozialordnung, Familie und Frauen, Staatsinstitut für Frühpädagogik. 2006. Der Bayerische Bildungs- und Erziehungsplan für Kinder in Tageseinrichtungen bis zur Einschulung. Weinheim, Basel: Beltz.

Beber, Katinka. 2008. «Dialogische Arbeit mit Familien.»In Professionalisierung der Frühpädagogik: Perspektiven, Entwicklungen, Herausforderungen, hrsg. v. Hilde von Balluseck, 177-182. Opladen, Farmington Hills: Barbara Budrich.

BMFSFJ. 2012. "Weitere 1.000 «Schwerpunkt-Kitas Sprache und Integration».» 30.3.2012. http://www.bmfsfj.de/BMFSFJ/kinder-und-jugend,did=185538.html.

Börsenverein des Deutschen Buchhandels in Zusammenarbeit mit der Arbeitsgemeinschaft von Jugendbuchverlagen e.V. 2007. Kinder- und Jugendbücher. Marktpotenzial, Käuferstrukturen und Präferenzen unterschiedlicher Lebenswelten. Frankfurt am Main.

Büchner, Peter und Anna Brake. 2006. Transmission von Bildung und Kultur in Mehrgenerationenfamilien im komplexen Netz gesellschaftlicher Anerkennungsbeziehungen: Resümee und Ausblick. Wiesbaden: VS-Verlag für Sozialwissenschaften.

Familie.de. "Kinder im Vorschulalter richtig erziehen.» Letzter Zugriff 25.4.2012. http://www.familie.de/kind/erziehung/artikel/kinder-im-vorschulalter-richtig -erziehen/ schwankendes-verhalten-im-vorschulalter/.

Fthenakis, Wassilios Emmanuel, Hrsg. 2006. Elementarpädagogik nach PISA. Wie aus Kindertagesstätten Bildungseinrichtungen werden können. 5. Aufl. Freiburg: Herder.

Fthenakis, Wassilios Emmanuel und Martin Textor, Hrsg. 2000. Pädagogische Ansätze im Kindergarten. Weinheim: Beltz.

Fuhs, Burkhard. 1997. "Spielen oder gleich «was Richtiges machen`? Zur sozialen Bedeutung des Spielens im Kindesalter.» In Spiele der Kinder: Interdisziplinäre Annäherungen, hrsg. v. Erich Renner, Sabine Riemann, Ilona K. Schneider u. Thomas Trautmann, 19-41. Weinheim: Dt. Studien Verlag.

Laewen, Hans-Joachim. 2007 (2002). «Was Bildung und Erziehung in Kindertageseinrichtungen bedeuten können.» In Forscher, Künstler, Konstrukteure: Werkstattbuch zum Bildungsauftrag von Kindertageseinrichtungen, hrsg. v. HansJoachim Laewen u. Beate Andres, 33-69. Berlin: Cornelsen. 
Laewen, Hans-Joachim und Beate Andres, Hrsg. 2007 (2002). Forscher, Künstler, Konstrukteure: Werkstattbuch zum Bildungsauftrag von Kindertageseinrichtungen. Berlin: Cornelsen.

Lange, Andreas. 2010. «Familie, Medien und die Tradierung sozialer Ungleichheiten.»In Medien. Bildung. Soziale Ungleichheit: Differenzen und Ressourcen im Mediengebrauch Jugendlicher, hrsg. v. Helga Theunert, 99-118. München: kopaed.

Mayr, Toni und Michaela Ulich. 2003. «Die Engagiertheit von Kindern: Zur systematischen Reflexion von Bildungsprozessen in Kindertageseinrichtungen.» In Elementarpädagogik nach PISA. Wie aus Kindertagesstätten Bildungseinrichtungen werden können, hrsg. v. Wassilios E. Fthenakis, 169-189. Freiburg: Herder.

Mollenhauer, Klaus. 1996. Grundfragen ästhetischer Bildung: Theoretische und empirische Befunde zur ästhetischen Erfahrung von Kindern. Weinheim, München: Juventa.

Niesyto, Horst. 2010. «Medienpädagogik: Milieusensible Förderung und Medienkompetenz.» In Medien. Bildung. Soziale Ungleichheit: Differenzen und Ressourcen im Mediengebrauch Jugendlicher, hrsg. v. Helga Theunert, 147-162. München: kopaed.

Nieswiodek-Martin, Ellen. 2006. Kinder in der Mediengesellschaft: Fernsehen, Computer und Erziehung. 2. Aufl. Holzgerlingen: SCM Hänssler.

Paus-Haase, Ingrid, Hrsg. 1997. Neue Helden für die Kleinen. Münster: LIT.

Sanktjohanser, Angelika. 2005. «Bildungskongress. Kindergeld ist eine Fehlinvestition.» FOCUS Online. Letzter Zugriff 25.4.2012. http://www.focus.de/wissen /bildungskongress_aid_100852.html.

Schäfer, Gerd E. 2005. Bildungsprozesse im Kindesalter. Selbstbildung, Erfahrung und Lernen in der frühen Kindheit. 3. Aufl. Weinheim: Juventa.

Singer, Wolf. 2003. «Was kann ein Mensch wann lernen? Ein Beitrag aus Sicht der Hirnforschung.» In Elementarpädagogik nach PISA: Wie aus Kindertagesstätten Bildungseinrichtungen werden können, hrsg. v. Wassilios E. Fthenakis, 6777. Freiburg: Herder.

Six, Ulrike und Roland Gimmler. 2007. Die Förderung von Medienkompetenz im Kindergarten: Eine empirische Studie zu Bedingungen und Handlungsformen der Medienerziehung. Berlin: Vistas.

Spanhel, Dieter. 2006. Handbuch Medienpädagogik. Medienerziehung Bd. 3. Stuttgart: Klett-Cotta.

SÜDWESTMETALL. Verband der Metall- und Elektroindustrie Baden-Württemberg e.V. Letzter Zugriff 23.4.2012. http://www.suedwestmetall.de/swm/web.nsf/id /pa_de_fruehfoerderung.html. 
Süss, Daniel, Lampert, Claudia und Christine W. Wijnen. 2010. Medienpädagogik: Ein Studienbuch zur Einführung. Wiesbaden: VS Verlag für Sozialwissenschaften.

Tietze, Wolfgang, Melnaie Bolz, Katja Grenner, Daena Schlecht und Beate Wellner. 2007. Krippen-Skala (KRIPS-R): Feststellung und Unterstützung pädagogischer Qualität in Krippen. (Deutsche Fassung der Infant/Toddler Environment Rating Scale Revised Edition). Berlin: Cornelsen.

Theunert, Helga, Hrsg. 2007. Medienkinder von Geburt an: Medienaneignung in den ersten sechs Lebensjahren. München: kopaed.

Theunert, Helga. 2009. "Kinder.»In Grundbegriffe Medienpädagogik - Praxis, hrsg. v. Bernd Schorb, Günther Anfang u. Kathrin Demmler, 142-144. München: kopaed.

Theunert, Helga, Hrsg. 2010. Medien. Bildung. Soziale Ungleichheit: Differenzen und Ressourcen im Mediengebrauch Jugendlicher. München: kopaed.

Thiele, Jens, Hrsg. 2007. Neue Impulse der Bilderbuchforschung. Baltmannsweiler: Schneider Verlag Hohengehren.

Thüringer Ministerium für Bildung, Wissenschaft und Kultur, Hrsg. 2010. Thüringer Bildungsplan für Kinder bis 10 Jahre. Weimar: verlag das netz. Letzter Zugriff 25.4.2012. Erfurt. www.tmbwk. de. https://www.thueringen.de/imperia/md/content/tmbwk/kindergarten/bildungsplan/th_bp_2011.pdf.

Winnicott, Donald W. 2005. Playing and Reality. London: Routledge. 